\title{
On the norms of circulant and $r$-circulant matrices with the hyperharmonic Fibonacci numbers
}

Naim Tuglu' and Can Kızılateş ${ }^{2 *}$

\section{"Correspondence:}

cankizilates@gmail.com

${ }^{2}$ Department of Mathematics,

Bülent Ecevit University, Zonguldak,

67100, Turkey

Full list of author information is

available at the end of the article

\begin{abstract}
In this paper, we study norms of circulant and $r$-circulant matrices involving harmonic Fibonacci and hyperharmonic Fibonacci numbers. We obtain inequalities by using matrix norms.
\end{abstract}

MSC: Primary 15A60; secondary 15B05; 11B39

Keywords: hyperharmonic Fibonacci number; r-Circulant matrix; matrix norm

\section{Introduction}

The circulant and $r$-circulant matrices have a connection to signal processing, probability, numerical analysis, coding theory, and many other areas. An $n \times n$ matrix $C_{r}$ is called an $r$-circulant matrix defined as follows:

$$
C_{r}=\left(\begin{array}{cccccc}
c_{0} & c_{1} & c_{2} & \cdots & c_{n-2} & c_{n-1} \\
r c_{n-1} & c_{0} & c_{1} & \cdots & c_{n-3} & c_{n-2} \\
r c_{n-2} & r c_{n-1} & c_{0} & \cdots & c_{n-4} & c_{n-3} \\
\vdots & \vdots & \vdots & & \vdots & \vdots \\
r c_{1} & r c_{2} & r c_{3} & \cdots & r c_{n-1} & c_{0}
\end{array}\right) .
$$

Since the matrix $C_{r}$ is determined by its row elements and $r$, we denote $C_{r}=\operatorname{Circ}\left(c_{0}, c_{1}, c_{2}\right.$, $\left.\ldots, c_{n-1}\right)$. In particular for $r=1$

$$
C=\left(\begin{array}{cccccc}
c_{0} & c_{1} & c_{2} & \cdots & c_{n-2} & c_{n-1} \\
c_{n-1} & c_{0} & c_{1} & \cdots & c_{n-3} & c_{n-2} \\
c_{n-2} & c_{n-1} & c_{0} & \cdots & c_{n-4} & c_{n-3} \\
\vdots & \vdots & \vdots & & \vdots & \vdots \\
c_{1} & c_{2} & c_{3} & \cdots & c_{n-1} & c_{0}
\end{array}\right)
$$

is called a circulant matrix and we denote it for brevity by $C=\operatorname{Circ}\left(c_{0}, c_{1}, c_{2}, \ldots, c_{n-1}\right)$. The eigenvalues of $C$ are

(c) 2015 Tuglu and Kızılateş. This article is distributed under the terms of the Creative Commons Attribution 4.0 International License (http://creativecommons.org/licenses/by/4.0/), which permits unrestricted use, distribution, and reproduction in any medium, provided you give appropriate credit to the original author(s) and the source, provide a link to the Creative Commons license, and indicate if changes were made. 


$$
\lambda_{j}=\sum_{i=0}^{n-1} c_{i}\left(w^{j}\right)^{i}
$$

where $w=e^{\frac{2 \pi i}{n}}$ and $i=\sqrt{-1}$.

Many authors have investigated the norms of circulant and $r$-circulant matrices. In [1], Solak studied the lower and upper bounds for the spectral norms of circulant matrices with classical Fibonacci and Lucas numbers entries. In [2], Kocer et al. obtained norms of circulant and semicirculant matrices with Horadams numbers. In [3], Zhou et al. gave the spectral norms of circulant-type matrices involving binomial coefficients and harmonic numbers. In [4], Zhou calculated spectral norms for circulant matrices with binomial coefficients combined with Fibonacci and Lucas number entries. In [5], Shen and Cen have given upper and lower bounds for the spectral norms of $r$-circulant matrices with classical Fibonacci and Lucas number entries. In [6], Bahşı and Solak computed the spectral norms of circulant and $r$-circulant matrices with the hyper-Fibonacci and hyper-Lucas numbers. In [7], Jiang and Zhou studied spectral norms of even-order $r$-circulant matrices.

Motivated by the above papers, we compute the spectral norms and Euclidean norm of circulant and $r$-circulant matrices with the harmonic and hyperharmonic Fibonacci entries. The scheme of this paper is as follows. In Section 2, we present some definitions, preliminaries, and lemmas related to our study. In Section 3, we calculate spectral norms of circulant matrix with harmonic Fibonacci entries. Moreover, we obtain the Euclidean norms of $r$-circulant matrices and give lower and upper bounds for the spectral norms of $r$-circulant matrices with harmonic and hyperharmonic Fibonacci entries.

\section{Preliminaries}

The Fibonacci numbers $F_{n}$ are defined by the following recurrence relation for $n \geq 1$ :

$$
F_{n+1}=F_{n}+F_{n-1}
$$

where $F_{0}=0, F_{1}=1$. In [8], the authors investigated the finite sum of the reciprocals of Fibonacci numbers,

$$
\mathbb{F}_{n}=\sum_{k=1}^{n} \frac{1}{F_{k}}
$$

which are called harmonic Fibonacci numbers. Then they gave a combinatoric identity related to harmonic Fibonacci numbers as follows:

$$
\sum_{k=0}^{n-1} F_{k-1} \mathbb{F}_{k}=F_{n} \mathbb{F}_{n}-n
$$

Moreover, in [8], they defined hyperharmonic Fibonacci numbers for $n, r \geq 1$

$$
\mathbb{F}_{n}^{(r)}=\sum_{k=1}^{n} \mathbb{F}_{k}^{(r-1)},
$$

where $\mathbb{F}_{n}^{(0)}=\frac{1}{F_{n}}$ and $\mathbb{F}_{0}=0$. At this point, we give some definitions and lemmas related to our study. 
Definition 1 Let $A=\left(a_{i j}\right)$ be any $m \times n$ matrix. The Euclidean norm of matrix $A$ is

$$
\|A\|_{E}=\sqrt{\left(\sum_{i=1}^{m} \sum_{j=1}^{n}\left|a_{i j}\right|^{2}\right) .}
$$

Definition 2 Let $A=\left(a_{i j}\right)$ be any $m \times n$ matrix. The spectral norm of matrix $A$ is

$$
\|A\|_{2}=\sqrt{\max _{1 \leq i \leq n} \lambda_{i}\left(A^{H} A\right)},
$$

where $\lambda_{i}\left(A^{H} A\right)$ is an eigenvalue of $A^{H} A$ and $A^{H}$ is the conjugate transpose of matrix $A$.

Then the following inequalities hold for the Euclidean norm and the spectral norm:

$$
\begin{aligned}
& \frac{1}{\sqrt{n}}\|A\|_{E} \leq\|A\|_{2} \leq\|A\|_{E}, \\
& \|A\|_{2} \leq\|A\|_{E} \leq \sqrt{n}\|A\|_{2} .
\end{aligned}
$$

Lemma 1 [9] Let $A$ and $B$ be two $m \times n$ matrices. Then we have

$$
\|A \circ B\|_{2} \leq\|A\|_{2}\|B\|_{2},
$$

where $A \circ B$ is the Hadamard product of $A$ and $B$.

Lemma 2 [9] Let $A$ and $B$ be two $n \times m$ matrices. We have

$$
\|A \circ B\|_{2} \leq r_{1}(A) c_{1}(B)
$$

where

$$
\begin{aligned}
& r_{1}(A)=\max _{1 \leq i \leq m} \sqrt{\sum_{j=1}^{n}\left|a_{i j}\right|^{2}}, \\
& c_{1}(B)=\max _{1 \leq j \leq n} \sqrt{\sum_{i=1}^{m}\left|b_{i j}\right|^{2} .}
\end{aligned}
$$

Definition 3 [10] The difference operator of $f(x)$ is defined as

$$
\Delta f(x)=f(x+1)-f(x)
$$

Definition 4 [10] A function $f(x)$ with the property that $\Delta f(x)=g(x)$ is called the antidifference operator of $g(x)$.

Lemma 3 [10] If $\Delta f(x)=g(x)$, then

$$
\sum_{a}^{b} g(x) \delta_{x}=\sum_{x=a}^{b-1} g(x)=f(b)-f(a) .
$$


Lemma 4 [10] We have

$$
\sum_{a}^{b} u(x) \Delta v(x) \delta_{x}=\left.u(x) v(x)\right|_{a} ^{b+1}-\sum_{a}^{b} v(x+1) \Delta u(x) \delta_{x} .
$$

Lemma 5 [10] For $m \neq-1$ we have

$$
\sum x^{\underline{m}} \delta_{x}=\frac{x^{\underline{m+1}}}{m+1}
$$

where $x^{\underline{m}}=x(x-1)(x-2) \cdots(x-m+1)$.

\section{Main results}

Theorem 1 [8] Let $C_{1}=\operatorname{Circ}\left(\mathbb{F}_{0}, \mathbb{F}_{1}, \mathbb{F}_{2}, \ldots, \mathbb{F}_{n-1}\right)$ be an $n \times n$ circulant matrix. The spectral norm of $C_{1}$ is

$$
\left\|C_{1}\right\|_{2}=n \mathbb{F}_{n}-\sum_{k=0}^{n-1} \frac{k+1}{F_{k+1}} .
$$

Theorem 2 [8] Let $C^{(k)}=\operatorname{Circ}\left(\mathbb{F}_{0}^{(k)}, \mathbb{F}_{1}^{(k)}, \mathbb{F}_{2}^{(k)}, \ldots, \mathbb{F}_{n-1}^{(k)}\right)$ be an $n \times n$ circulant matrix. The spectral norm of $C^{(k)}$ is

$$
\left\|C^{(k)}\right\|_{2}=\mathbb{F}_{n-1}^{(k+1)}
$$

\section{Theorem 3 Let}

$$
C=\operatorname{Circ}\left(F_{-1} \mathbb{F}_{0}, F_{0} \mathbb{F}_{1}, \ldots, F_{n-2} \mathbb{F}_{n-1}\right)
$$

be an $n \times n$ circulant matrix. Then the spectral norm of the matrix $C$ is

$$
\|C\|_{2}=F_{n} \mathbb{F}_{n}-n .
$$

Proof Since $C$ is a circulant matrix, from (1), for all $t=0,1, \ldots, s-1$,

$$
\lambda_{t}(C)=\sum_{i=0}^{s-1} F_{i-1} \mathbb{F}_{i}\left(w^{t}\right)^{i}
$$

Then, for $t=0$,

$$
\lambda_{0}(C)=\sum_{i=0}^{s-1} F_{i-1} \mathbb{F}_{i}
$$

and from (2), $\lambda_{0}(C)=F_{n} \mathbb{F}_{n}-n$. Hence, for $1 \leq m \leq n-1$, we have

$$
\left|\lambda_{m}\right|=\left|\sum_{i=0}^{s-1} F_{i-1} \mathbb{F}_{i}\left(w^{t}\right)^{i}\right| \leq\left|\sum_{i=0}^{s-1} F_{i-1} \mathbb{F}_{i}\right|\left|\left(w^{t}\right)^{i}\right| \leq \sum_{i=0}^{s-1} F_{i-1} \mathbb{F}_{i} .
$$


Since $C$ is a normal matrix, we have

$$
\|C\|_{2}=\max _{0 \leq m \leq n-1}\left|\lambda_{m}\right|
$$

From (7), (8), (9), and (2), we have

$$
\|C\|_{2}=F_{n} \mathbb{F}_{n}-n
$$

\section{Corollary 1 We have}

$$
\sqrt{\sum_{k=0}^{n-1} F_{k-1}^{2} \mathbb{F}_{n}^{2}} \leq F_{n} \mathbb{F}_{n}-n \leq \sqrt{n \sum_{k=0}^{n-1} F_{k-1}^{2} \mathbb{F}_{n}^{2}}
$$

Proof The proof is trivial from Definition 1 and the relation between the Euclidean norm and the spectral norm in (3).

Theorem 4 Let

$$
C_{r}^{(k)}=\operatorname{Circ}\left(\mathbb{F}_{0}^{(k)}, \mathbb{F}_{1}^{(k)}, \ldots, \mathbb{F}_{n-1}^{(k)}\right)
$$

be an $n \times n$ r-circulant matrix. The Euclidean norm of $C_{r}^{(k)}$ is

$$
\begin{aligned}
\left\|C_{r}^{(k)}\right\|_{E}= & {\left[\frac{n}{2}\left(n+1+(n-1)|r|^{2}\right)\left(\mathbb{F}_{n}^{(k)}\right)^{2}\right.} \\
& \left.-\frac{1}{2} \sum_{s=0}^{n-1}(s+1)\left(2 n+s\left(|r|^{2}-1\right)\right)\left(\mathbb{F}_{s+1}^{(k-1)}+2 \mathbb{F}_{s}^{(k)}\right) \mathbb{F}_{s+1}^{(k-1)}\right]^{\frac{1}{2}} .
\end{aligned}
$$

Proof From the definition of the Euclidean norm we have

$$
\begin{aligned}
\left\|C_{r}^{(k)}\right\|_{E} & =\left[\sum_{s=0}^{n-1}(n-s)\left(\mathbb{F}_{s}^{(k)}\right)^{2}+\sum_{s=0}^{n-1} s|r|^{2}\left(\mathbb{F}_{s}^{(k)}\right)^{2}\right]^{\frac{1}{2}} \\
& =\left[\sum_{s=0}^{n-1}\left(n+s\left(|r|^{2}-1\right)\right)\left(\mathbb{F}_{s}^{(k)}\right)^{2}\right]^{\frac{1}{2}} .
\end{aligned}
$$

Now we will use the property of the difference operator in Lemma 4. Let $u(s)=\left(\mathbb{F}_{s}^{(k)}\right)^{2}$ and $\Delta v(s)=n+s\left(|r|^{2}-1\right)$. Then using the definition of the hyperharmonic Fibonacci numbers we obtain $\Delta u(s)=\mathbb{F}_{s+1}^{(k-1)}\left(\mathbb{F}_{s+1}^{(k-1)}+2 \mathbb{F}_{s}^{(k)}\right)$ and $v(s)=n s+\frac{s^{2}}{2}\left(|r|^{2}-1\right)$. By using (5), we have

$$
\begin{aligned}
\left\|C_{r}^{(k)}\right\|_{E}= & {\left[\frac{n}{2}\left(n+1+(n-1)|r|^{2}\right)\left(\mathbb{F}_{n}^{(k)}\right)^{2}\right.} \\
& \left.-\frac{1}{2} \sum_{s=0}^{n-1}(s+1)\left(2 n+s\left(|r|^{2}-1\right)\right)\left(\mathbb{F}_{s+1}^{(k-1)}+2 \mathbb{F}_{s}^{(k)}\right) \mathbb{F}_{s+1}^{(k-1)}\right]^{\frac{1}{2}} .
\end{aligned}
$$


Corollary 2 Let $C_{r}=\operatorname{Circ}\left(\mathbb{F}_{0}, \mathbb{F}_{1}, \ldots, \mathbb{F}_{n-1}\right)$ be an $n \times n$-circulant matrix. The Euclidean norm of $C_{r}$ is

$$
\begin{aligned}
\left\|C_{r}\right\|_{E}= & {\left[\left(n^{2}+\frac{n^{\underline{2}}}{2}\left(|r|^{2}-1\right)\right) \mathbb{F}_{n}^{2}\right.} \\
& \left.-\sum_{s=0}^{n-1}\left(n(s+1)+\frac{(s+1)^{2}}{2}\left(|r|^{2}-1\right)\right)\left(2 \mathbb{F}_{s}+\frac{1}{F_{s+1}}\right) \frac{1}{F_{s+1}}\right]^{\frac{1}{2}} .
\end{aligned}
$$

Proof It is clear that the proof can be completed if we take $k=1$ in Theorem 4 .

Corollary 3 [8] Let $C_{1}=\operatorname{Circ}\left(\mathbb{F}_{0}, \mathbb{F}_{1}, \ldots, \mathbb{F}_{n-1}\right)$ be an $n \times n$ matrix. The Euclidean norm is

$$
\left\|C_{1}\right\|_{E}=\left[n^{2} \mathbb{F}_{n}^{2}-n \sum_{k=0}^{n-1} \frac{k+1}{F_{k+1}}\left(2 \mathbb{F}_{k}+\frac{1}{F_{k+1}}\right)\right]^{\frac{1}{2}} .
$$

Proof It is easily seen that the proof can be completed if we take $k=r=1$ in Theorem 4 .

Now we give upper and lower bounds for the spectral norms of $r$-circulant matrices.

Theorem 5 Let $C_{r}^{(k)}=\operatorname{Circ}\left(\mathbb{F}_{0}^{(k)}, \mathbb{F}_{1}^{(k)}, \ldots, \mathbb{F}_{n-1}^{(k)}\right)$ be an $n \times n$ r-circulant matrix.

(i) If $|r| \geq 1$, then

$$
\frac{1}{\sqrt{n}} \mathbb{F}_{n-1}^{(k+1)} \leq\left\|C_{r}^{(k)}\right\|_{2} \leq|r| \sqrt{n-1} \mathbb{F}_{n-1}^{(k+1)}
$$

(ii) If $|r|<1$, then

$$
\frac{|r|}{\sqrt{n}} \mathbb{F}_{n-1}^{(k+1)} \leq\left\|C_{r}^{(k)}\right\|_{2} \leq \sqrt{n-1} \mathbb{F}_{n-1}^{(k+1)} .
$$

Proof Since we have the matrix

$$
C_{r}^{(k)}=\left(\begin{array}{cccccc}
\mathbb{F}_{0}^{(k)} & \mathbb{F}_{1}^{(k)} & \mathbb{F}_{2}^{(k)} & \cdots & \mathbb{F}_{n-2}^{(k)} & \mathbb{F}_{n-1}^{(k)} \\
r \mathbb{F}_{n-1}^{(k)} & \mathbb{F}_{0}^{(k)} & \mathbb{F}_{1}^{(k)} & \cdots & \mathbb{F}_{n-3}^{(k)} & \mathbb{F}_{n-2}^{(k)} \\
\vdots & \vdots & \vdots & & \vdots & \vdots \\
r \mathbb{F}_{2}^{(k)} & r \mathbb{F}_{3}^{(k)} & r \mathbb{F}_{4}^{(k)} & \cdots & \mathbb{F}_{0}^{(k)} & \mathbb{F}_{1}^{(k)} \\
r \mathbb{F}_{1}^{(k)} & r \mathbb{F}_{2}^{(k)} & r \mathbb{F}_{3}^{(k)} & \cdots & r \mathbb{F}_{n-1}^{(k)} & \mathbb{F}_{0}^{(k)}
\end{array}\right),
$$

we have

$$
\left\|C_{r}^{(k)}\right\|_{E}=\sqrt{\sum_{s=0}^{n-1}(n-s)\left(\mathbb{F}_{s}^{(k)}\right)^{2}+\sum_{s=0}^{n-1} s|r|^{2}\left(\mathbb{F}_{s}^{(k)}\right)^{2}} .
$$

(i) In [8], for the sum of the squares of hyperharmonic Fibonacci numbers, we have

$$
\frac{1}{\sqrt{n}} \mathbb{F}_{n-1}^{(r+1)} \leq \sqrt{\sum_{k=0}^{n-1}\left(\mathbb{F}_{k}^{(r)}\right)^{2}} \leq \mathbb{F}_{n-1}^{(r+1)} .
$$


Since $|r| \geq 1$ and by (11), we have

$$
\left\|C_{r}^{(k)}\right\|_{E} \geq \sqrt{\sum_{s=0}^{n-1}(n-s)\left(\mathbb{F}_{s}^{(k)}\right)^{2}+\sum_{s=0}^{n-1} s\left(\mathbb{F}_{s}^{(k)}\right)^{2}} \geq \sqrt{n \sum_{s=0}^{n-1}\left(\mathbb{F}_{s}^{(k)}\right)^{2}} \geq \mathbb{F}_{n-1}^{(k+1)}
$$

From (3)

$$
\frac{1}{\sqrt{n}} \mathbb{F}_{n-1}^{(k+1)} \leq\left\|C_{r}^{(k)}\right\|_{2}
$$

On the other hand, let the matrices $A$ and $B$ be defined by

$$
A=\left(\begin{array}{cccccc}
\mathbb{F}_{0}^{(k)} & 1 & 1 & \cdots & 1 & 1 \\
r & \mathbb{F}_{0}^{(k)} & 1 & \cdots & 1 & 1 \\
\vdots & \vdots & \vdots & & \vdots & \vdots \\
r & r & r & \cdots & \mathbb{F}_{0}^{(k)} & 1 \\
r & r & r & \cdots & r & \mathbb{F}_{0}^{(k)}
\end{array}\right)
$$

and

$$
B=\left(\begin{array}{cccccc}
\mathbb{F}_{0}^{(k)} & \mathbb{F}_{1}^{(k)} & \mathbb{F}_{2}^{(k)} & \ldots & \mathbb{F}_{n-2}^{(k)} & \mathbb{F}_{n-1}^{(k)} \\
\mathbb{F}_{n-1}^{(k)} & \mathbb{F}_{0}^{(k)} & \mathbb{F}_{1}^{(k)} & \ldots & \mathbb{F}_{n-3}^{(k)} & \mathbb{F}_{n-2}^{(k)} \\
\vdots & \vdots & \vdots & & \vdots & \vdots \\
\mathbb{F}_{2}^{(k)} & \mathbb{F}_{3}^{(k)} & \mathbb{F}_{4}^{(k)} & \ldots & \mathbb{F}_{0}^{(k)} & \mathbb{F}_{1}^{(k)} \\
\mathbb{F}_{1}^{(k)} & \mathbb{F}_{2}^{(k)} & \mathbb{F}_{3}^{(k)} & \ldots & \mathbb{F}_{n-1}^{(k)} & \mathbb{F}_{0}^{(k)}
\end{array}\right)
$$

That is, $C_{r}^{(k)}=A \circ B$. Then we obtain

$$
r_{1}(A)=\max _{1 \leq i \leq n} \sqrt{\sum_{j=1}^{n}\left|a_{i j}\right|^{2}}=\sqrt{\sum_{j=1}^{n}\left|a_{n j}\right|^{2}}=\sqrt{(n-1)|r|^{2}}
$$

and

$$
c_{1}(B)=\max _{1 \leq j \leq n} \sqrt{\sum_{i=1}^{n}\left|b_{i j}\right|^{2}}=\sqrt{\sum_{i=1}^{n}\left|b_{i n}\right|^{2}}=\sqrt{\sum_{s=0}^{n-1}\left(\mathbb{F}_{s}^{(k)}\right)^{2}} .
$$

Hence, from (11) and Lemma 1, we have

$$
\left\|C_{r}^{(k)}\right\|_{2} \leq|r| \sqrt{n-1} \mathbb{F}_{n-1}^{(k+1)}
$$

Thus, we have

$$
\frac{1}{\sqrt{n}} \mathbb{F}_{n-1}^{(k+1)} \leq\left\|C_{r}^{(k)}\right\|_{2} \leq|r| \sqrt{n-1} \mathbb{F}_{n-1}^{(k+1)}
$$


(ii) From $|r|<1$ and from (11), we have

$$
\begin{aligned}
\left\|C_{r}^{(k)}\right\|_{E} & =\sqrt{\sum_{s=0}^{n-1}(n-s)\left(\mathbb{F}_{s}^{(k)}\right)^{2}+\sum_{s=0}^{n-1} s|r|^{2}\left(\mathbb{F}_{s}^{(k)}\right)^{2}} \\
& \geq \sqrt{\sum_{s=0}^{n-1}(n-s)|r|^{2}\left(\mathbb{F}_{s}^{(k)}\right)^{2}+\sum_{s=0}^{n-1} s|r|^{2}\left(\mathbb{F}_{s}^{(k)}\right)^{2}} \\
& =|r| \sqrt{n \sum_{s=0}^{n-1}\left(\mathbb{F}_{s}^{(k)}\right)^{2}} \\
& \geq|r| \mathbb{F}_{n-1}^{(k+1)} .
\end{aligned}
$$

From (3),

$$
\frac{|r|}{\sqrt{n}} \mathbb{F}_{n-1}^{(k+1)} \leq\left\|C_{r}^{(k)}\right\|_{2}
$$

On the other hand, let the matrices $A$ and $B$ be defined by

$$
A=\left(\begin{array}{cccccc}
\mathbb{F}_{0}^{(k)} & 1 & 1 & \cdots & 1 & 1 \\
r & \mathbb{F}_{0}^{(k)} & 1 & \cdots & 1 & 1 \\
\vdots & \vdots & \vdots & & \vdots & \vdots \\
r & r & r & \cdots & \mathbb{F}_{0}^{(k)} & 1 \\
r & r & r & \cdots & r & \mathbb{F}_{0}^{(k)}
\end{array}\right)
$$

and

$$
B=\left(\begin{array}{cccccc}
\mathbb{F}_{0}^{(k)} & \mathbb{F}_{1}^{(k)} & \mathbb{F}_{2}^{(k)} & \ldots & \mathbb{F}_{n-2}^{(k)} & \mathbb{F}_{n-1}^{(k)} \\
\mathbb{F}_{n-1}^{(k)} & \mathbb{F}_{0}^{(k)} & \mathbb{F}_{1}^{(k)} & \cdots & \mathbb{F}_{n-3}^{(k)} & \mathbb{F}_{n-2}^{(k)} \\
\vdots & \vdots & \vdots & & \vdots & \vdots \\
\mathbb{F}_{2}^{(k)} & \mathbb{F}_{3}^{(k)} & \mathbb{F}_{4}^{(k)} & \ldots & \mathbb{F}_{0}^{(k)} & \mathbb{F}_{1}^{(k)} \\
\mathbb{F}_{1}^{(k)} & \mathbb{F}_{2}^{(k)} & \mathbb{F}_{3}^{(k)} & \cdots & \mathbb{F}_{n-1}^{(k)} & \mathbb{F}_{0}^{(k)}
\end{array}\right) .
$$

Thus $C_{r}^{(k)}=A \circ B$. Then we obtain

$$
r_{1}(A)=\max _{1 \leq i \leq n} \sqrt{\sum_{j=1}^{n}\left|a_{i j}\right|^{2}}=\sqrt{\left(\mathbb{F}_{0}^{(k)}\right)^{2}+n-1}=\sqrt{n-1}
$$

and

$$
c_{1}(B)=\max _{1 \leq j \leq n} \sqrt{\sum_{i=1}^{n}\left|b_{i j}\right|^{2}}=\sqrt{\sum_{s=0}^{n-1}\left(\mathbb{F}_{s}^{(k)}\right)^{2}} .
$$

Therefore, from (11) and Lemma 1, we have

$$
\left\|C_{r}^{(k)}\right\|_{2} \leq \sqrt{n-1} \mathbb{F}_{n-1}^{(k+1)}
$$


Thus, we have

$$
\frac{|r|}{\sqrt{n}} \mathbb{F}_{n-1}^{(k+1)} \leq\left\|C_{r}^{(k)}\right\|_{2} \leq \sqrt{n-1} \mathbb{F}_{n-1}^{(k+1)} .
$$

Corollary 4 Let $C_{r}=\operatorname{Circ}\left(\mathbb{F}_{0}, \mathbb{F}_{1}, \ldots, \mathbb{F}_{n-1}\right)$ be an $n \times n$ r-circulant matrix.

(i) If $|r| \geq 1$, then

$$
\frac{1}{\sqrt{n}} \mathbb{F}_{n-1}^{(2)} \leq\left\|C_{r}\right\|_{2} \leq|r| \sqrt{n-1} \mathbb{F}_{n-1}^{(2)}
$$

(ii) If $|r|<1$, then

$$
\frac{|r|}{\sqrt{n}} \mathbb{F}_{n-1}^{(2)} \leq\left\|C_{r}\right\|_{2} \leq \sqrt{n-1} \mathbb{F}_{n-1}^{(2)} .
$$

Proof It is easily seen that the proof can be completed if we take $k=1$ in Theorem 5 .

\section{Numerical examples}

In this section, we present some numerical examples by using Maple 11.

Example 1 Let $C=\operatorname{Circ}\left(F_{-1} \mathbb{F}_{0}, F_{0} \mathbb{F}_{1}, \ldots, F_{n-2} \mathbb{F}_{n-1}\right)$ be as in (6). We obtain the spectral norms of some $n \times n C$ matrices, with the aid of Theorem 3 (see Table 1 ).

Example 2 Let $C_{r}^{(k)}=\operatorname{Circ}\left(\mathbb{F}_{0}^{(k)}, \mathbb{F}_{1}^{(k)}, \ldots, \mathbb{F}_{4}^{(k)}\right)$ be $5 \times 5 r$-circulant matrix as in (10). We obtain Euclidean norms of $C_{r}^{(k)}$ for some values of $r$ and $k$, with the aid of Theorem 4 (see Table 2).

Example 3 Let $C_{r}^{(k)}=\operatorname{Circ}\left(\mathbb{F}_{0}^{(k)}, \mathbb{F}_{1}^{(k)}, \ldots, \mathbb{F}_{4}^{(k)}\right)$ be $5 \times 5 r$-circulant matrix as in (10). We obtain some lower and upper bounds for the spectral norms of $C_{r}^{(k)}$ for some values of $r$ and $k$, with the aid of Theorem 5 (see Tables 3 and 4).

Table 1 Spectral norms of $C$

\begin{tabular}{ll}
\hline $\boldsymbol{n}$ & $\| \boldsymbol{C}_{\mathbf{2}}$ \\
\hline$n=5$ & $0.1016666667 \times 10^{2}$ \\
$n=10$ & $0.1731757972 \times 10^{3}$ \\
$n=50$ & $0.4228842484 \times 10^{11}$ \\
$n=100$ & $0.1190154990 \times 10^{22}$ \\
$n=500$ & $0.4684460937 \times 10^{105}$ \\
$n=1,000$ & $0.1460426641 \times 10^{210}$ \\
\hline
\end{tabular}

Table 2 Euclidean norms of $C_{r}^{(k)}$ for $n=5$

\begin{tabular}{lllll}
\hline $\boldsymbol{k} / \boldsymbol{r}$ & $\boldsymbol{k}=\mathbf{1}$ & $\boldsymbol{k}=\mathbf{2}$ & $\boldsymbol{k}=\mathbf{3}$ & $\boldsymbol{k}=\mathbf{4}$ \\
\hline$r=-2$ & $\frac{\sqrt{9,935}}{6}$ & $\frac{\sqrt{61,598}}{6}$ & $\frac{\sqrt{246,743}}{6}$ & $\frac{\sqrt{755,966}}{6}$ \\
$r=-0.5$ & $\frac{\sqrt{7,415}}{12}$ & $\frac{\sqrt{37,127}}{12}$ & $\frac{\sqrt{136,007}}{12}$ & $\frac{\sqrt{397,559}}{12}$ \\
$r=0.1$ & $\frac{\sqrt{133,655}}{60}$ & $\frac{\sqrt{593,351}}{60}$ & $\frac{\sqrt{2,038,631}}{60}$ & $\frac{\sqrt{5,736,887}}{60}$ \\
$r=0.9$ & $\frac{\sqrt{306,055}}{60}$ & $\frac{\sqrt{1,709,431}}{60}$ & $\frac{\sqrt{6,577,111}}{60}$ & $\frac{\sqrt{19,743,847}}{60}$ \\
$r=1$ & $\frac{\sqrt{3,470}}{6}$ & $\frac{\sqrt{19,745}}{6}$ & $\frac{5 \sqrt{3,062}}{6}$ & $\frac{\sqrt{230,705}}{6}$ \\
$r=1.1$ & $\frac{\sqrt{392,255}}{60}$ & $\frac{\sqrt{2,267,471}}{60}$ & $\frac{\sqrt{8,846,351}}{60}$ & $\frac{\sqrt{26,747,327}}{60}$ \\
$r=10$ & $\frac{\sqrt{216,815}}{6}$ & $\frac{\sqrt{1,400,894}}{6}$ & $\frac{\sqrt{5,692,919}}{6}$ & $\frac{\sqrt{17,564,318}}{6}$ \\
\hline
\end{tabular}


Table 3 Some lower and upper bounds for the spectral norms of $C_{r}^{(k)}$ for $n=5$ and $|r| \geq 1$

\begin{tabular}{llllc}
\hline & $|\boldsymbol{r}| \geq \mathbf{1}$ & $\frac{\mathbf{1}}{\sqrt{\boldsymbol{n}}} \mathbb{F}_{\boldsymbol{n}-\mathbf{1}}^{(\boldsymbol{k}+\mathbf{1})}$ & $\| \boldsymbol{C}_{\boldsymbol{r}}^{(\boldsymbol{k})} \boldsymbol{\|}_{\mathbf{2}}$ & $|\boldsymbol{r}| \sqrt{\boldsymbol{n - 1}} \mathbb{F}_{\boldsymbol{n}-\mathbf{1}}^{(\boldsymbol{k}+\mathbf{1})}$ \\
\hline$k=1$ & $r=-2$ & 3.726779962 & 13.07393997 & 33.33333333 \\
& $r=1$ & 3.726779962 & 8.333333332 & 16.66666667 \\
& $r=1.1$ & 3.726779962 & 6.187213310 & 18.33333333 \\
$k=2$ & $r=-2$ & 7.975309119 & 29.94984421 & 71.33333333 \\
& $r=1$ & 7.975309119 & 17.83333333 & 35.66666667 \\
& $r=1.1$ & 7.975309119 & 19.01179206 & 39.23333333 \\
$k=3$ & $r=-2$ & 14.45990625 & 56.50736302 & 129.3333333 \\
& $r=1$ & 14.45990625 & 32.33333334 & 64.66666667 \\
$k=4$ & $r=1.1$ & 14.45990625 & 34.60097132 & 71.13333333 \\
& $r=-2$ & 23.62778496 & 94.70523788 & 211.3333333 \\
& $r=1$ & 23.62778496 & 52.83333332 & 105.6666667 \\
& $r=1.1$ & 23.62778496 & 56.68238740 & 116.2333333 \\
\hline
\end{tabular}

Table 4 Some lower and upper bounds for the spectral norms of $C_{r}^{(k)}$ for $n=5$ and $|r|<1$

\begin{tabular}{|c|c|c|c|c|}
\hline & $|r|<1$ & $\frac{|r|}{\sqrt{n}} \mathbb{F}_{n-1}^{(k+1)}$ & $\left\|C_{r}^{(k)}\right\|_{2}$ & $\sqrt{n-1} \mathbb{F}_{n-1}^{(k+1)}$ \\
\hline \multirow[t]{3}{*}{$k=1$} & $r=-0.5$ & 1.863389981 & 6.051069352 & 16.66666667 \\
\hline & $r=0.1$ & 0.372677996 & 5.912862964 & 16.66666667 \\
\hline & $r=0.9$ & 3.354101966 & 7.874554252 & 16.66666667 \\
\hline \multirow[t]{3}{*}{$k=2$} & $r=-0.5$ & 3.987654558 & 13.14607243 & 35.66666667 \\
\hline & $r=0.1$ & 0.797530912 & 12.64953828 & 35.66666667 \\
\hline & $r=0.9$ & 7.177778206 & 16.74610810 & 35.66666667 \\
\hline \multirow[t]{3}{*}{$k=3$} & $r=-0.5$ & 7.229953125 & 24.39979647 & 64.66666667 \\
\hline & $r=0.1$ & 1.445990625 & 23.46328510 & 64.66666667 \\
\hline & $r=0.9$ & 13.01391563 & 30.26866878 & 64.66666667 \\
\hline \multirow[t]{3}{*}{$k=4$} & $r=-0.5$ & 11.81389248 & 40.74250279 & 105.6666667 \\
\hline & $r=0.1$ & 2.362778496 & 39.32798158 & 105.66666667 \\
\hline & $r=0.9$ & 21.26500646 & 49.37728355 & 105.66666667 \\
\hline
\end{tabular}

\section{Competing interests}

The authors declare that they have no competing interests.

\section{Authors' contributions}

Each of the authors, NT, and CK, contributed to each part of this work equally and read and approved the final version of the manuscript.

\section{Author details}

'Department of Mathematics, Gazi University, Teknikokullar, Ankara, 06500, Turkey. ${ }^{2}$ Department of Mathematics, Bülent Ecevit University, Zonguldak, 67100, Turkey.

\section{Acknowledgements}

The authors are grateful to two anonymous referees and the associate editor for their careful reading, helpful comments, and constructive suggestions, which improved the presentation of the results.

Received: 12 June 2015 Accepted: 5 August 2015 Published online: 20 August 2015

\section{References}

1. Solak, S: On the norms of circulant matrices with the Fibonacci and Lucas numbers. Appl. Math. Comput. 160, 125-132 (2005)

2. Kocer, EG, Mansour, T, Tuglu, N: Norms of circulant and semicirculant matrices with Horadams numbers. Ars Comb. $85,353-359(2007)$

3. Zhou, J, Chen, X, Jiang, Z: The explicit identities for spectral norms of circulant-type matrices involving binomial coefficients and harmonic numbers. Math. Probl. Eng. 2014, Article ID 518913 (2014)

4. Zhou, J: The identical estimates of spectral norms for circulant matrices with binomial coefficients combined with Fibonacci numbers and Lucas numbers entries. J. Funct. Spaces Appl. 2014, Article ID 672398 (2014)

5. Shen, SQ, Cen, JM: On the bounds for the norms of $r$-circulant matrices with Fibonacci and Lucas numbers. Appl. Math. Comput. 216, 2891-2897 (2010)

6. Bahşı, M, Solak, S: On the norms of $r$-circulant matrices with the hyper-Fibonacci and Lucas numbers. J. Math. Inequal. 8, 693-705 (2014)

7. Jiang, Z, Zhou, J: A note on spectral norms of even-order r-circulant matrices. Appl. Math. Comput. 250, 368-371 (2015)

8. Tuglu, N, Kizilates, C, Kesim, S: On the harmonic and hyperharmonic Fibonacci numbers. arXiv:1505.04284 
9. Horn, RA, Johnson, CR: Topics in Matrix Analysis. Cambridge University Press, Cambridge (1991)

10. Graham, RL, Knuth, DE, Patashnik, O: Concrete Mathematics, 2nd edn. Addison-Wesley, Reading (1994)

Submit your manuscript to a SpringerOpen ${ }^{\odot}$ journal and benefit from:

- Convenient online submission

Rigorous peer review

- Immediate publication on acceptance

- Open access: articles freely available online

- High visibility within the field

- Retaining the copyright to your article

Submit your next manuscript at $\boldsymbol{s p r i n g e r o p e n . c o m ~}$ 